\title{
The 6.5-m MMT Telescope: Status and Plans for the Future
}

\author{
G. Grant Williams ${ }^{\mathrm{a}}$, R. Ortiz ${ }^{\mathrm{a}}$, W. Goble ${ }^{\mathrm{a}}$, and J. D. Gibson ${ }^{\mathrm{a}}$ \\ ${ }^{a}$ MMT Observatory, P.O. Box 210065, University of Arizona, Tucson, AZ 85721-0065
}

\begin{abstract}
The MMT Observatory, a joint venture of the Smithsonian Institution and the University of Arizona, operates the 6.5-m MMT telescope on the summit of Mount Hopkins approximately 45 miles south of Tucson, AZ. The upgraded telescope has been in routine operation for nearly fifteen years and, as such, is a very reliable and productive general purpose astronomical instrument. The telescope can be configured with one of three secondary mirrors that feed more than ten instruments at the Cassegrain focus. In this paper we provide an overview of the telescope, its current capabilities, and its performance. We will review the existing suite of instruments and their different modes of operation. We will describe some of the general operations challenges and strategies for the Observatory. Finally, we will discuss plans for the near-term future including technical upgrades, new instrumentation and routine queue operation of MMIRS and Binospec.
\end{abstract}

Keywords: MMTO, Observatory Operations

\section{INTRODUCTION}

The MMT Observatory (MMTO) is operated under contract to serve the research requirements of astronomers at the University of Arizona Observatories (UAO) and the Smithsonian Astrophysical Observatory (SAO), a member of the Harvard-Smithsonian Center for Astrophysics (CfA). The MMT has a rich history as an innovative and productive astronomical facility. The combination of its large aperture, excellent seeing, ${ }^{1}$ multiple secondary mirrors, and versatile suite of instrumentation make the MMT a very powerful tool for a wide range of astronomical research.

The MMT is located on the summit of Mount Hopkins, approximately 45 miles south of Tucson, AZ. The telescope is part of the Fred Lawrence Whipple Observatory (FLWO) but operates independently because of the $\mathrm{SAO} / \mathrm{UA}$ partnership. MMTO staff are responsible for maintaining and operating everything inside the facilities at the summit and FLWO staff support the telescope's physical plant and infrastructure. MMTO, in turn, provides assistance to FLWO in the areas of communications and general engineering. Similarly, the MMTO turns to UA and SAO for technical assistance on occasion and the expertise developed by the staff is shared with the parent organizations. UA hosts staff offices and laboratories in Tucson and also supports MMTO administrative functions through its business infrastructure. The Smithsonian Institution provides facilities and site infrastructure support through the Smithsonian Office of Engineering and Operations (OFEO) and Smithsonian Office of Facilities Management \& Reliability (OFMR).

The original Multiple Mirror Telescope consisted of six 1.8-m mirrors on a common mount. The light from the six mirrors was combined and fed to a common Cassegrain focus. With an effective collecting area of a 4.5-m telescope, it became the third largest telescope in the world when it was dedicated in 1979. For more details on the history of the MMT see Ref. 2 and the MMT website, http://mmto.org/.

The 4.5-m MMT was shutdown in March 1998 to install a new 6.5-m telescope on the same mount in the slightly modified enclosure. This upgrade was enabled by technological advances pioneered by the Steward Observatory mirror lab (see Refs. 3 \& 4). At the time of its casting in 1992 the f/1.25 6.5-m borosilicate honeycomb mirror was the largest monolithic mirror that had ever been made. The converted telescope recorded first light on May 17, 2000 and became fully operational after the successful in-situ aluminization of the primary mirror in late 2001. Routine operation of the $6.5-\mathrm{m}$ telescope has now been ongoing for nearly 15 years.

Send correspondence to G. G. W.

E-mail: ggwilli@mmto.org, Telephone: 1.520.621.1558

Ground-based and Airborne Telescopes VI, edited by Helen J. Hall, Roberto Gilmozzi, Heather K. Marshall, Proc. of SPIE Vol. 9906, 99060V · C 2016 SPIE · CCC code: 0277-786X/16/\$18 · doi: 10.1117/12.2233777 
The MMT's primary mirror is positioned with six hardpoints and its weight is supported by 104 pneumatic actuators (48 dual axis, 52 single axis, and 4 cross laterals). The actuators are also used in the active optics system to bend the primary to correct for low order wavefront errors. To keep the mirror isothermal and at the ambient nighttime air temperature it is thermally controlled with a conditioned forced air ventilation system. ${ }^{5-7}$

\section{TELESCOPE AND INSTRUMENT CONFIGURATIONS}

The 6.5-m MMT telescope is a classical Cassegrain design that can be configured with three different secondary mirrors that provide final focal ratios of $\mathrm{f} / 5, \mathrm{f} / 9$ and $\mathrm{f} / 15$. The detailed optical specifications can be found in Ref. 8. The $\mathrm{f} / 5$ secondary can be used with a wide field corrector that can be configured for imaging (30' flat focal surface) or spectroscopy with a ADC (60' curved focal surface). ${ }^{9}$ The f/15 secondary was the world's first thin shell adaptive secondary mirror (ASM). Its 1.8-mm thick shell is deformed at $550 \mathrm{~Hz}$ by 336 voice coil actuators acting on rear surface magnets to correct for wavefront aberrations introduced by the Earth's atmosphere. ${ }^{10,11}$

Table 1. Characteristics of the MMT optics.

\begin{tabular}{|c|c|c|c|c|c|c|}
\hline Mirror & Glass & $\begin{array}{c}\text { Clear Diameter } \\
(\mathrm{mm})\end{array}$ & $\begin{array}{c}\text { Overall Diameter } \\
(\mathrm{mm})\end{array}$ & $\begin{array}{c}\text { Edge Thick. } \\
(\mathrm{mm})\end{array}$ & $\begin{array}{c}\text { Center Thick. } \\
(\mathrm{mm})\end{array}$ & $\begin{array}{c}\text { Weight } \\
(\mathrm{kg})\end{array}$ \\
\hline Primary & Ohara E6 & 6502 & 6512 & 711 & 391 & 7735 \\
\hline $\mathrm{f} / 5$ & Zerodur & 1692 & 1715 & 133 & 206 & 288 \\
\hline $\mathrm{f} / 9$ & Schott Tempax & 1007 & 1022 & 152 & 152 & 77 \\
\hline f/15 & Zeordur & 640 & 640 & 1.8 & 1.8 & - \\
\hline
\end{tabular}

Each secondary feeds a number of science instruments that are all mounted on a derotator at the Cassegrain focus. Although there is a Nasmyth platform, there is no tertiary mirror or Nasmyth instrumentation. Figures 1 \& 2 show the MMT instruments that are currently available for imaging and spectroscopy, respectively. The plots show field-of-view and resolving power together with wavelength coverage color coded and line style coded by secondary. Comprehensive overviews of the MMT instrumentation suite can be found in Refs. 12,13. Some of the previously available instruments are no longer offered because they've been decommissioned or transferred to another observatory.

The adaptive secondary mirror saw first light in 2002 and has been operating for more than 14 years. The system's age, a reduced number of spares, and an opportunity to provide prototype electronics for GMT drove a decision to consider upgrading the system soon. With the science goal of characterizing exoplanets and planetary systems in formation, an NSF MSIP proposal entitled "MMT Adaptive optics exoPlanet characterization System (MAPS)" was submitted in 2015 to upgrade the system. There were several proposed improvements including a pyramid wavefront sensor with an NIR/visible camera as well as upgrades to the ARIES instrument. The proposal was not fully fund but seed funds will be provided to upgrade the ASM electronics and recoat the mirror. The system will be taken off line in early 2017 for 18 months to complete the upgrade and recalibrate the ASM.

The MMT and Magellan Infrared Spectrograph (MMIRS) ${ }^{14}$ is an near infrared imager and multi-object spectrograph that was commissioned at the MMT in 2009. However, following commissioning it was shipped to Magellan where it was used for six years. In mid 2015 MMIRS was returned to the MMT providing a powerful bright time capability. The field-of-view for imaging is $6.9^{\prime} \times 6.9^{\prime}$ and for slit mask spectroscopy is reduced to $4.0^{\prime} \times 6.9^{\prime}$. There are multiple grism/filter combinations which provided resolving powers of 800 to 3000 (with a $0.4^{\prime \prime}$ slit). Multiple $420^{\prime \prime}$ long slits with widths ranging between $0.2^{\prime \prime}$ and $2.4^{\prime \prime}$ are also available. The sensitivity of the instrument was recently improved with the upgrade from a Hawaii2 to a Hawaii2-RG detector. ${ }^{15}$

Binospec $^{16}$ is a wide field optical imaging multi-object spectrograph with a long slit mode that is currently being built at SAO. It's scheduled to arrive at the MMT in early 2017. The instrument has two side-by-side $8^{\prime} \times 15^{\prime}$ fields-of-view that feed two sets of identical optics. Each slit mask can accommodate up to 150 slitlets. The instrument will use three gratings that provide resolving powers of 1350 to 9500 with a $1^{\prime \prime}$ slit. Since the 


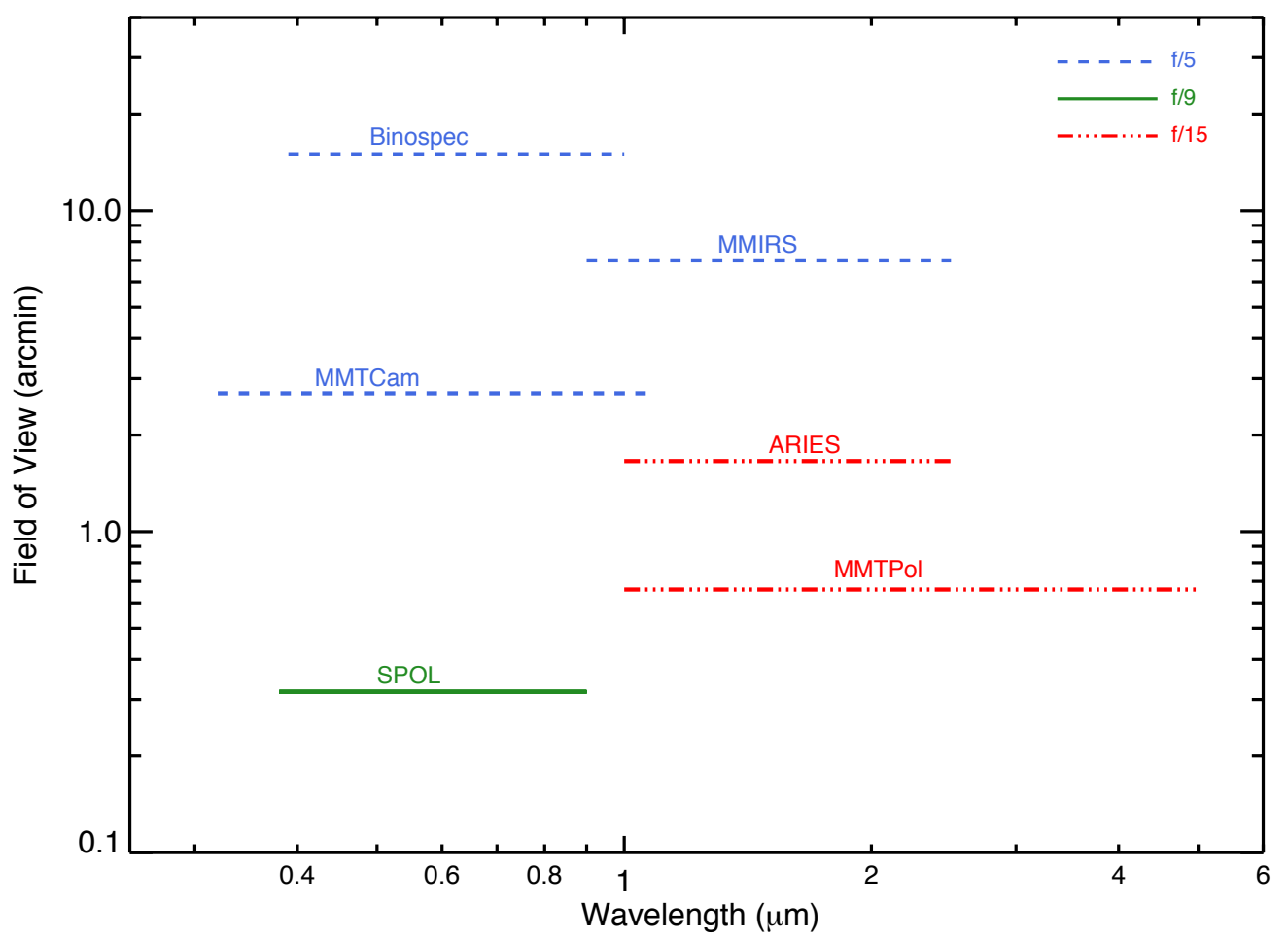

Figure 1. MMTO imaging instrumentation.

instrument provides a high throughput long slit mode, we anticipate that Binospec will be used for many of the projects that have, in the past, requested the workhorse single object spectrographs Blue Channel or Red Channel. Therefore, once Binospec arrives Red Channel will be decommissioned. Blue Channel will remain available because it provides coverage down to $3200 \AA$ and Binospec's throughput cuts off at $3900 \AA$.

Both Binsospec and MMIRS will join Hectospec as queue operated instruments. In the past, night assistants from FLWO have observed for the Hectospec and Hectochelle queues. However, in anticipation of many more queue scheduled nights with the arrival of MMIRS and Binospec MMTO has recently added two Queue Observers to the staff (see Fig. 3).

\section{PRIMARY MIRROR COATING}

The MMT operates eleven months of the year and shuts down for a four week annual maintenance period that coincides with poor weather associated with desert southwest's monsoon season. Every five years this shutdown period is used to re-coat the primary mirror. Prior to conversion of the MMT, a decision was made to coat the primary in-situ. The mirror cell itself together with steel rear covers and a bell jar front cover act as the vacuum vessel. The coating process that was used through the 2010 re-coating is provided in Ref. 17. A more detailed paper describing the process is also available at https://www.mmto.org/MMTpapers/pdfs/tm/tm03-8.pdf.

A significant amount of the preparation for the coating must wait until the telescope is taken off-line. Therefore, the very serial process typically takes $\sim 10$ weeks to complete. Because of the extra time required to coat the mirror, MMTO makes a strong effort toward maintaining the coating to keep the reflectivity high and the scattering low. This is accomplished with $\sim$ monthly $\mathrm{CO} 2$ "snow" cleanings and biannual contact mirror washes. 


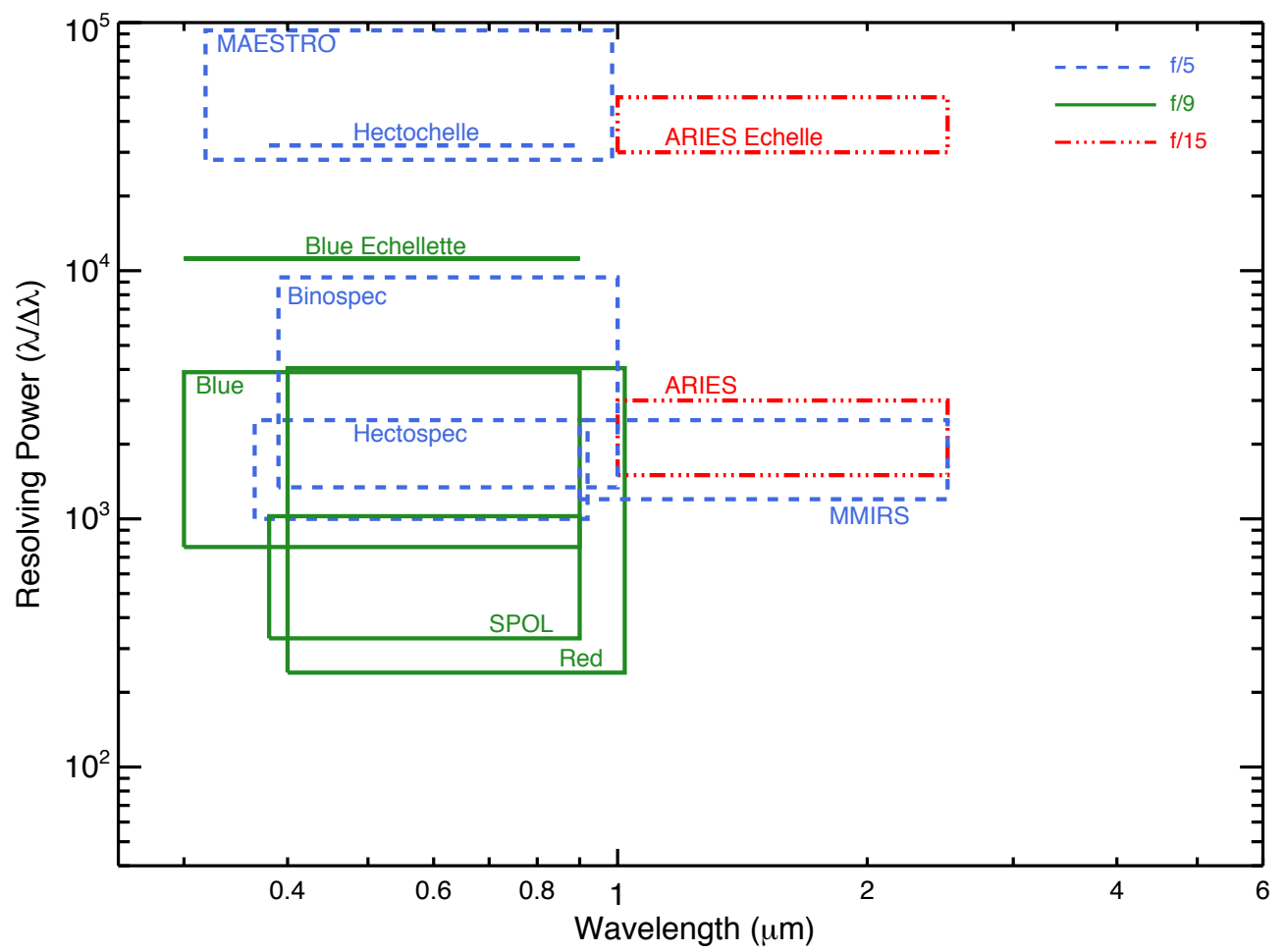

Figure 2. MMTO spectroscopic instrumentation.

Since conversion to the $6.5-\mathrm{m}$ telescope there have been five coatings of the primary mirror with mixed results. The first shot in 1999 resulted in a layer vaporized tungsten on top of the aluminum that provided poor reflectivity. The second shot in spring 2000 resulted in a coating that was contaminated by copper that evaporated from the power cables, once again providing a low but in this case usable reflectivity $(\sim 70 \%)$. The first fully successful shot occurred in November 2001. A subsequent shot in 2005 was also successful. However, in 2010 molten aluminum dripped from the filaments and splashed onto an aluminized mylar vacuum membrane that isolates the "dirty" back side from the "clean" front side. Mylar fumes were deposited with the aluminum resulting in a blemished area of the coating that extended to cover approximately $1 / 8$ of the mirror. The reflectivity in the blemished area was very wavelength dependent and as low as $\sim 40 \%$ (in the blue) in the core but increased significantly away from the core. Despite the blemish, the reflectivity was very good over the majority of the surface. In fact the reflectivity in the blemished area improved with every contact wash.

In order to avoid repeating the incident caused by dripping aluminum that occurred in 2010, the MMT's aluminization process was reevaluated and several improvements were identified. These improvements included but were not limited to (1) a new integrally wound tungsten and aluminum filament, (2) an automated filament heating process, and (3) a protective shield to cover the mylar.

Many months were spent upgrading and characterizing the new system. Subsets of the system were tested at the Steward Observatory's Sunnyside small optics coating facility. There were also three full scale tests performed in a setup at the FLWO basecamp. A description of the characterization of the new filament style is provided in Ref. 18 and a description of the software for the process is provided in Ref. 19. Details of the entire system and the full scale tests will be written up in a future publication. During shutdown 2016 the primary mirror will be recoated for the first time using the new process. 


\section{OBSERVATORY OPERATIONS}

\subsection{Organization Structure}

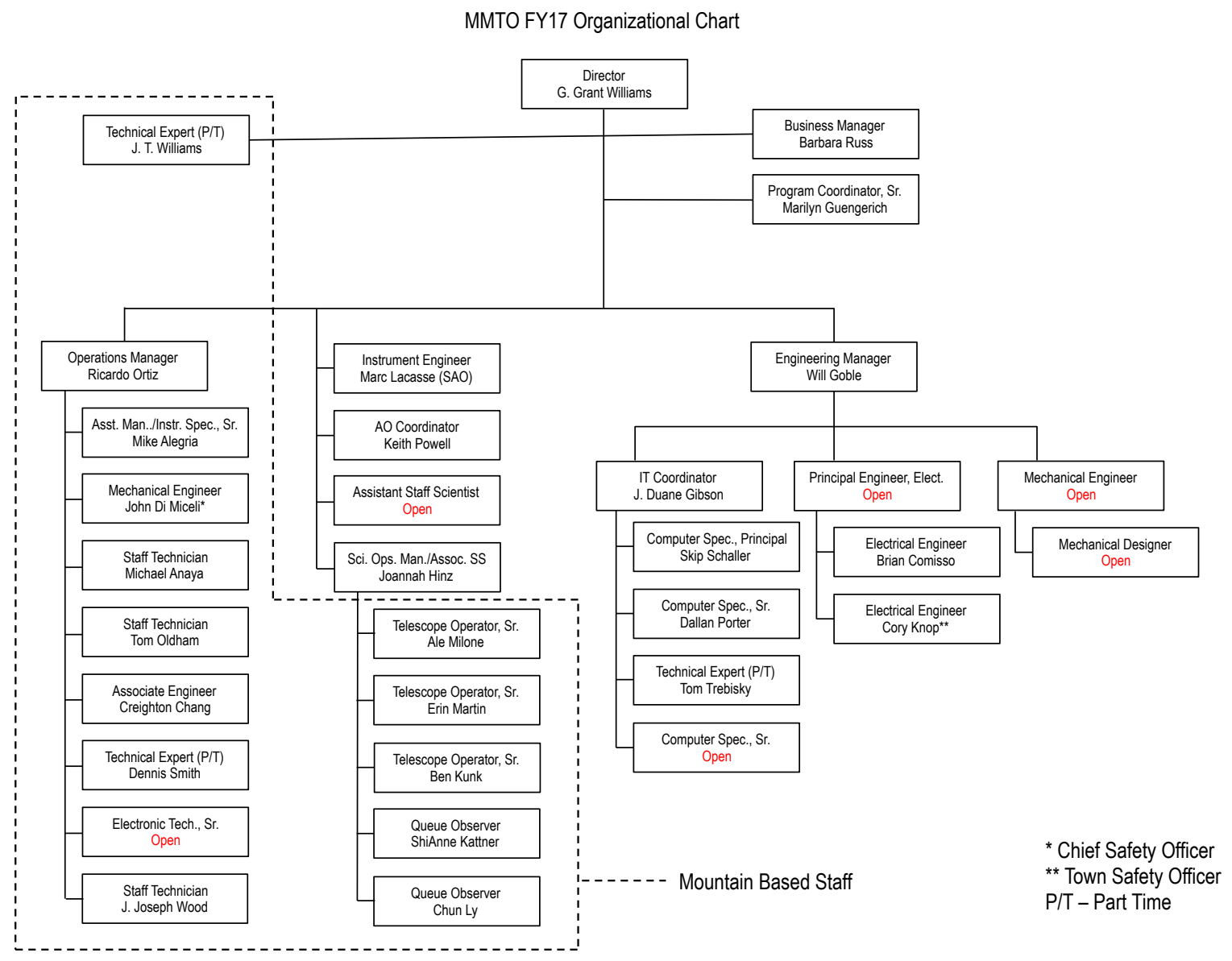

Figure 3. MMTO's FY17 Organizational Chart

The workload at the MMTO can be divided into operations, maintenance, and development, though there is considerable overlap among the three categories. Most MMTO staff members contribute to each of these areas. Figure 3 shows the FY17 Organizational Chart for the MMT Observatory. There are currently $\sim 27$ full-time and $\sim 3$ part time staff members split between the duty stations of "mountain" and "town".

The MMTO Director is fully responsible for the safe and efficient operation of the Observatory. Toward this end, the Director receives advice and assistance from an Operations Manager, an Engineering Manager, Staff Scientists, a Business Manager, and a Program Coordinator. Guided by general priorities approved by the MMTO Council, the Director establishes the overall plan for technical projects, oversees observatory operations, and paces and monitors progress.

The two Queue Observer positions are new to the organizational chart and therefore, because of budget constraints, it's unlikely that all of the open positions will be filled. In particular, it's likely that only one or two of the four positions of Computer Specialist, Sr., Mechanical Engineer, Mechanical Designer, and Electronic Technician position, will be filled.

\subsection{Operations Approach}

In order to enable a productive Observatory, the MMT staff strive to minimize technical down time. Figure 4 shows the annual time lost to technical issues during the last 13 years broken into the three categories of 
instrument, telescope and general facility. The positive trend toward a reduction in lost time is evident. During the last five years the average time lost to technical issues has been less than $3.2 \%$ per year. However, we have yet to reach a floor which is likely to be below $2 \%$. Our plans for the near future are to implement more quality control and preventative maintenance measures that will ensure the favorable trend continues. We will also work toward minimizing operational inefficiencies that impact shutter open time.

Our general strategy to minimizing lost time includes the following approach on weekdays and only a slightly different approach on weekends.

- Every staff member reads the nightly operators $\log$ as the first order of daily business. This is often done even before staff leave their homes so that they can plan for the day accordingly.

- Group leaders and senior staff meet in a daily tag-up meeting at 11:30 am. The purpose of this meeting is to address every issue in the previous nights operator/astronomer/AO log and service requests (SRs). The time of this meeting is late enough to allow staff to address issues before the meeting and therefore provide updates at the meeting but early enough to provide time after the meeting for major repair efforts and travel to the mountain should additional support be required.

- Ensure due diligence to checking out all issues indicated in the logs and SRs. It's not uncommon that an odd noise or event is a precursor to a more catastrophic failure. Since we dont know a priori which log items fall into this potentially failing status, we need to do due diligence to investigating them all.

- Work to reduce the number of unaddressed SRs to ensure that no issues fall through the cracks. The Assistant Operations Manager is responsible for regularly reviewing open requests and following up with staff on the status of the technical issues.

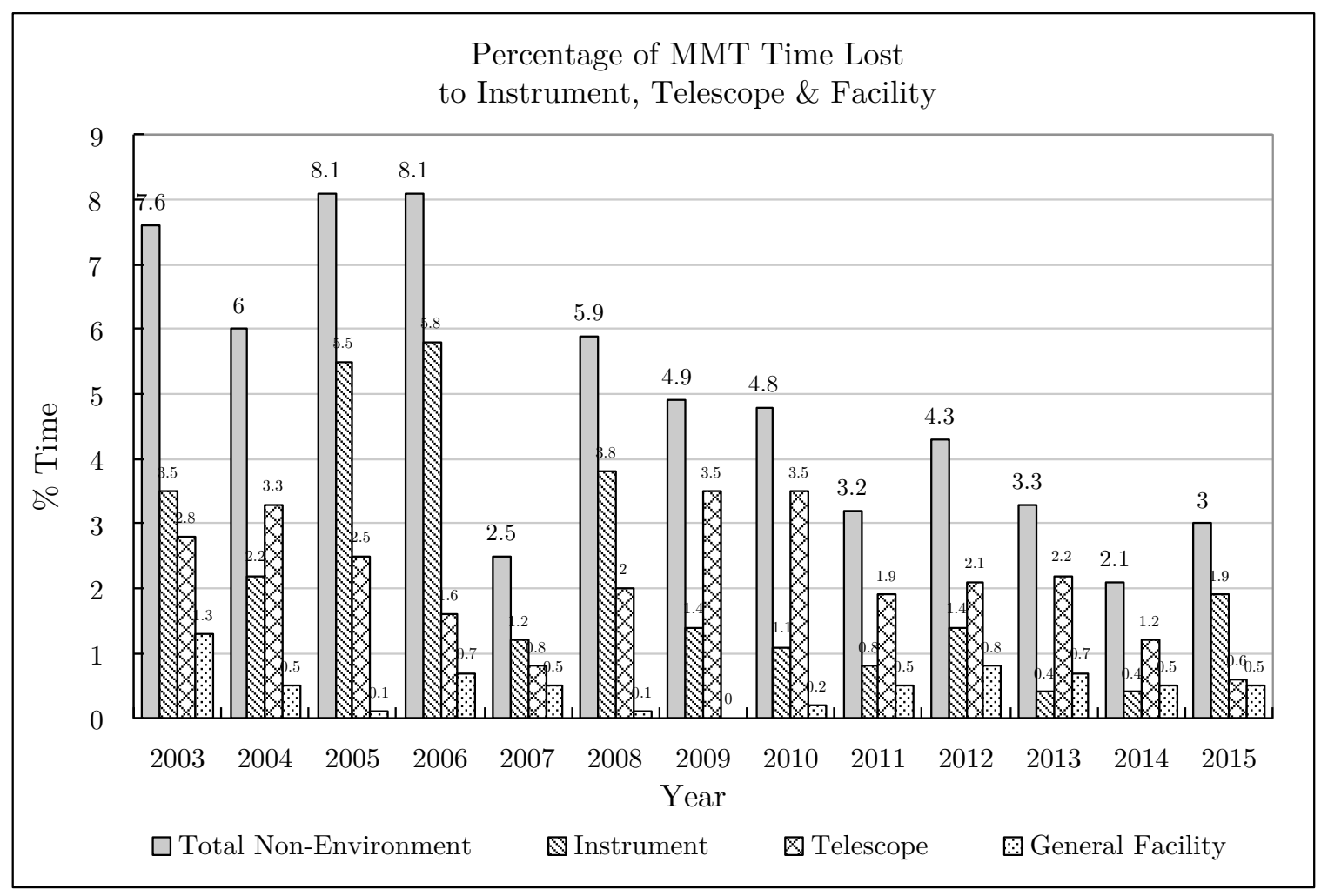

Figure 4. MMTO Annual Technical Down Time Statistics 
- Continuously improve procedures for mounting and removing instruments and secondaries. This will ultimately lower the probability of improper installation that could lead to lost time or safety incidents. It also includes adding critical steps to the procedures to confirm proper operation of any newly installed instrument or secondary.

- Document as much as possible. Good documentation will reduce troubleshooting time and avoid diversions from the actual issue.

- Follow a comprehensive preventative maintenance (PM) schedule. A basic electronic PM schedule that sends automated emails was implemented in 2015 and continues to be updated.

\subsection{Time Allocation \& Scheduling}

The available observing time for the MMTO has traditionally been divided among five groups: SAO scientists, UAO scientists, MMTO Director's discretionary time, visiting astronomers (NOAO Public Access), and maintenance and engineering $(\mathrm{M} \& \mathrm{E})$ time. The Public Access time was provided in exchange for instrumentation funding through NSF as part of the Telescope System Instrumentation Program (TSIP). The NSF/TSIP program no longer exists and the public access time was depleted in 2012. Currently the MMT observing time is apportioned as $44 \%$ each for SAO and UAO, $6 \%$ for Director's discretionary time, and $6 \%$ for M\&E.

The MMT observing time has, in the past, been allocated and scheduled on a trimester basis. However, starting in January 2013 the UAO time has been allocated on a semester basis. This difference in semester (UAO) versus trimester (SAO) allocations could result in difficulties in scheduling. However, the UAO TAC has split the semesters as Jan-Jul and Aug-Dec. With this seven/five month split and with summer shutdown taking up most of the month of August, the impact on scheduling has been minimized and it continues to be done three times a year. The three annual MMT scheduling periods are now January 1 - April 30, May 1 - July 31, and August 1 - December 31. Since August is part of trimester two for SAO and semester two for UAO, the end of August (after summer shutdown) will typically be scheduled in the third scheduling period.

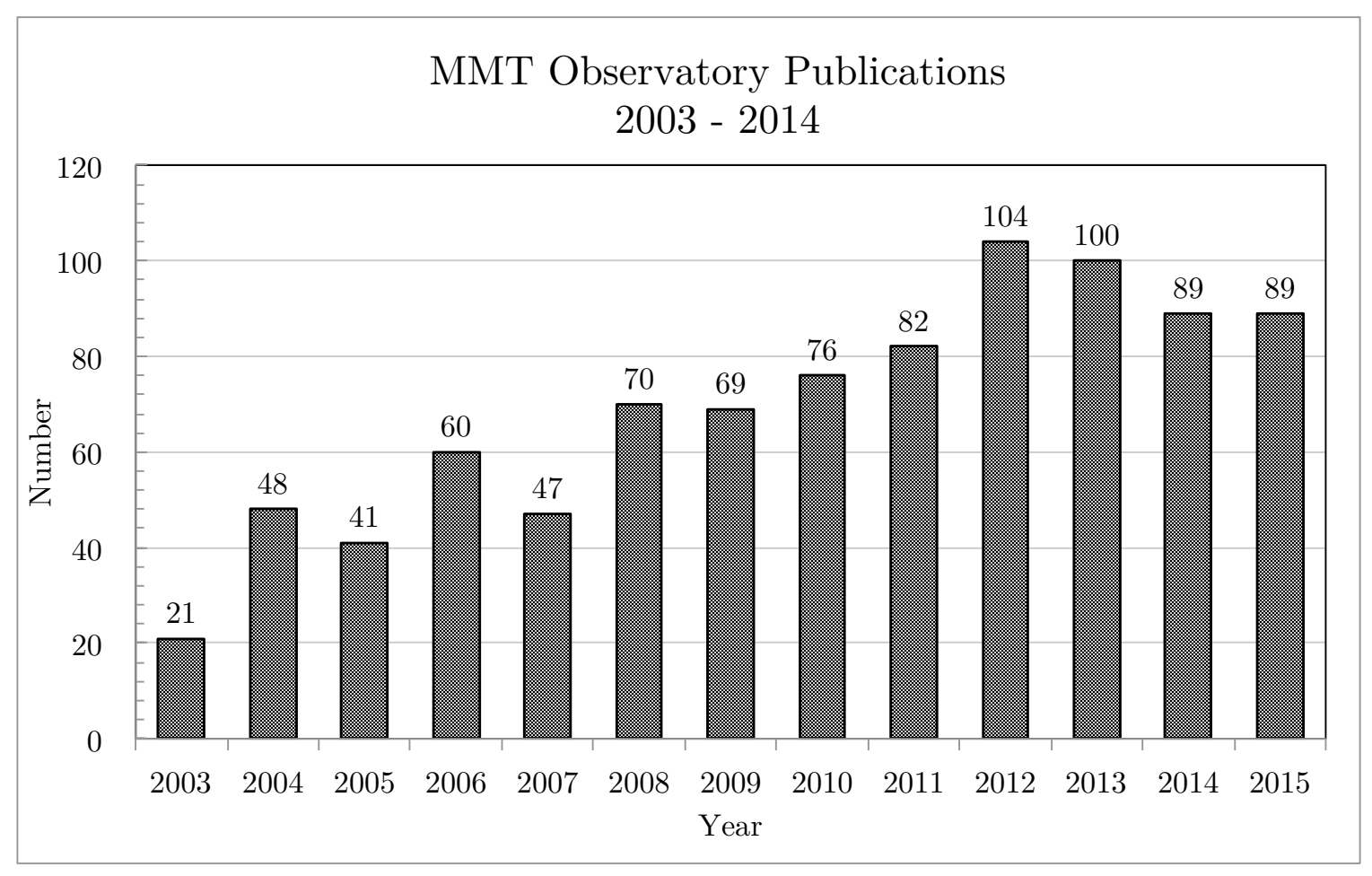

Figure 5. MMTO Based Publications 
Scheduling the MMT includes the following steps: a) the MMTO Director, with assistance from the Program Coordinator, Sr., determines the number of nights of each lunar phase for that trimester; b) the MMTO Director divides the time into the four categories listed above and distributes the result to the Time Allocation Committees (TACs); c) the UAO and SAO TACs rank their proposals and assign their share of nights; d) these programs, the MMTO Director's discretionary time, and M\&E are then scheduled in a meeting between the MMTO Director and representatives from SAO (generally only observing the process remotely) and UAO.

MMTO will soon implement a new integrated scheduling and program management system more commonly referred to as the "scheduler database". Development of the system was described in detail by Ref. 20. The basis of the database will be the telescope schedule with proposal information included as metadata. Several administrative tasks will be automated based on the information in the database, e.g. dorm reservations, observing forms, etc. During operations additional information from each night will be appended the database, e.g. targets observed, atmospheric conditions, etc. This system should significantly reduce some of the current administrative burden while at the same time enabling capabilities that would have been tedious as manual projects in the past.

\section{OBSERVATORY PRODUCTIVITY AND IMPACT}

Given that the MMTO staff and resources are limited we must prioritize our engineering activities to avoid over commitment. Prioritization of specific engineering activities often depends on several factors including safety, urgency, cost, demand, and potential return. We attempt to set the priorities to maximize the productivity and impact of the Observatory.

Observatories often use publication-based metrics to assess productivity and impact. These are usually measured by the number of publications and the number of citations to those publications, respectively. However, it's important to point out that, although publication based metrics are paramount, they are not the only metrics by which the productivity and the impact of the MMTO should be measured. For example, engineering accomplishments, which will usually (but not always) result in a publication, will often drive strategic plans for the MMTO and other future observatories. It's difficult to quantify these but it's important to acknowledge their benefit.

The level of performance and scientific productivity of the MMT remains very high. Figure 5 shows the annual publication statistics. The 89 MMTO based publications during the 2015 calendar year shares third ranking with 2014 and behind only the previous two years of 2012 and 2013. The MMTs productivity (number of publications) and impact (citations) are now compared to our peer observatories by D. Crabtree (see Refs. 21,22). During the 2008-2013 period, the MMTO was very competitive with its peers but more importantly the trends were extremely encouraging.

\section{ACKNOWLEDGMENTS}

The authors would like to acknowledge the hard work and dedication of the entire MMT Observatory staff. Observations reported here were obtained at the MMT Observatory, a joint facility of the University of Arizona and the Smithsonian Institution.

\section{REFERENCES}

[1] Gibson, J. D., Williams, G. G., and Trebisky, T., "Seeing trends from deployable Shack-Hartmann wavefront sensors, MMT Observatory, Arizona, USA," in [Ground-based and Airborne Telescopes IV], Proc. SPIE 8444, 844432 (Sept. 2012).

[2] Blanco, D., Alegria, M., Callahan, S., Clark, D., Comisso, B., Foltz, C. B., Gibson, J. D., Heller, C., James, R., Kindred, B., King, S., Knop, C., Lester, H., McAfee, J., Milone, A. A. E., Ortiz, R., Pickering, T. E., Ritz, P., Russ, B., Schmidt, G., Smith, D., Spencer, P., Trebisky, T., Van Horn, K., West, S. C., Wainwright, C., Williams, G., and Williams, J. T., "The new MMT," in [Ground-based Telescopes], Oschmann, Jr., J. M., ed., Proc. SPIE 5489, 300-311 (Oct. 2004). 
[3] Olbert, B. H., Angel, J. R. P., Hill, J. M., and Hinman, S. F., "Casting 6.5-meter mirrors for the MMT conversion and Magellan," in [Advanced Technology Optical Telescopes V], Stepp, L. M., ed., Proc. SPIE 2199, 144-155 (June 1994).

[4] Martin, H. M., Burge, J. H., Ketelsen, D. A., and West, S. C., "Fabrication of the 6.5-m primary mirror for the Multiple Mirror Telescope Conversion," in [Optical Telescopes of Today and Tomorrow], Ardeberg, A. L., ed., Proc. SPIE 2871, 399-404 (Mar. 1997).

[5] Cheng, A. Y. S. and Angel, J. R. P., "Steps toward 8m honeycomb mirrors. VIII - Design and demonstration of a system of thermal control," in [Advanced technology optical telescopes III], Barr, L. D., ed., Proc. SPIE 628, 536-544 (Jan. 1986).

[6] Williams, G. G., Gibson, J. D., Callahan, S., Blanco, D., Williams, J. T., and Spencer, P., "Performance and control of the MMT thermal system," in [Ground-based Telescopes], Oschmann, Jr., J. M., ed., Proc. SPIE 5489, 938-949 (Oct. 2004).

[7] Gibson, J. D., Porter, D., and Goble, W., "Automation and control of the MMT thermal system," in [Software and Cyberinfrastructure for Astronomy IV], Proc. SPIE 9913 (July 2016).

[8] Fabricant, D. G., McLeod, B., and West, S., "Optical specifications for the MMT conversion, Version 7." MMT Technical Memo, http://www.mmto.org/sites/default/files/mmt_conv7_2.pdf (Dec. 1999).

[9] Fata, R. G. and Fabricant, D. G., "Mounting large lenses in wide-field instruments for the converted MMT," in [Optical Astronomical Instrumentation], D’Odorico, S., ed., Proc. SPIE 3355, 275-284 (July 1998).

[10] Martin, H. M., Burge, J. H., Del Vecchio, C., Dettmann, L. R., Miller, S. M., Smith, B. K., and Wildi, F. P., "Optical fabrication of the MMT adaptive secondary mirror," in [Adaptive Optical Systems Technology], Wizinowich, P. L., ed., Proc. SPIE 4007, 502-507 (July 2000).

[11] Lloyd-Hart, M., Wildi, F. P., Martin, B., McGuire, P. C., Kenworthy, M. A., Johnson, R. L., Fitz-Patrick, B. C., Angeli, G. Z., Miller, S. M., and Angel, J. R. P., "Adaptive optics for the 6.5-m MMT," in [Adaptive Optical Systems Technology], Wizinowich, P. L., ed., Proc. SPIE 4007, 167-174 (July 2000).

[12] Hastie, M. and McLeod, B., "Comprehensive review of the converted MMT's instrument suite," in [Groundbased and Airborne Instrumentation for Astronomy II], Proc. SPIE 7014, 70140B (July 2008).

[13] Hastie, M. and Williams, G. G., "Instrumentation suite at the MMT Observatory," in [Ground-based and Airborne Instrumentation for Astronomy III], Proc. SPIE 7735, 773507 (July 2010).

[14] McLeod, B., Fabricant, D., Nystrom, G., McCracken, K., Amato, S., Bergner, H., Brown, W., Burke, M., Chilingarian, I., Conroy, M., Curley, D., Furesz, G., Geary, J., Hertz, E., Holwell, J., Matthews, A., Norton, T., Park, S., Roll, J., Zajac, J., Epps, H., and Martini, P., "MMT and Magellan Infrared Spectrograph," PASP 124, 1318-1335 (Dec. 2012).

[15] Chilingarian, I., Beletsky, Y., Moran, S., Brown, W., McLeod, B., and Fabricant, D., "Data Reduction Pipeline for the MMT and Magellan Infrared Spectrograph," PASP 127, 406-420 (Apr. 2015).

[16] Fabricant, D. G., Epps, H. W., Brown, W. L., Fata, R. G., and Mueller, M., "Development of Binospec and its optics," in [Instrument Design and Performance for Optical/Infrared Ground-based Telescopes], Iye, M. and Moorwood, A. F. M., eds., Proc. SPIE 4841, 1134-1144 (Mar. 2003).

[17] Clark, D., Kindred, W., and Williams, J. T., "In situ aluminization of the MMT 6.5m primary mirror," in [Society of Photo-Optical Instrumentation Engineers (SPIE) Conference Series], Proc. SPIE 6273, 627305 (June 2006).

[18] Goble, W. and Ortiz, R., "Characterization of an integrally wound tungsten and aluminum filament for use in the MMTO 6.5m primary mirror aluminization chamber," in [Advances in Optical and Mechanical Technologies for Telescopes and Instrumentation], Proc. SPIE 9912 (July 2016).

[19] Gibson, J. D., Clark, D., and Porter, D., "Software framework for the upcoming MMT Observatory primary mirror re-aluminization," in [Software and Cyberinfrastructure for Astronomy III], Proc. SPIE 9152, 91521K (July 2014).

[20] Porter, D., Gibson, J. D., and Williams, G. G., "An integrated scheduling and program management system," in [Observatory Operations: Strategies, Processes, and Systems IV], Proc. SPIE 8448, 844824 (Sept. 2012).

[21] Crabtree, D. R., "A bibliometric analysis of observatory publications for the period 2008-2012," in [Observatory Operations: Strategies, Processes, and Systems V], Proc. SPIE 9149, 91490A (July 2014). 
[22] Crabtree, D. R., "A bibliometric analysis of observatory publications for the period 2009-2013," in [Observatory Operations: Strategies, Processes, and Systems VI], Proc. SPIE 9910 (July 2016). 